\title{
RESSECÇÃO DE METÁSTASE HEPÁTICA DE SARCOMAS DE PARTES MOLES
}

\author{
LIVER RESECTION FOR METASTATIC SOFT TISSUE SARCOMAS
}

\author{
Rinaldo Gonçalves da Silva ${ }^{1}$; Rubens Kesley Siqueira de Paiva²; \\ Carlos Eduardo Rodrigues Santos- TCBC-RJ² ; Eduardo Linhares Riello de Melo- TCBC-RJ²; \\ Jurandir de Almeida Dias- TCBC-RJ ${ }^{3}$; Guilherme Pinto Bravo Neto, TCBC-RJ ${ }^{4}$
}

\begin{abstract}
RESUMO: Objetivo: Avaliar os resultados do tratamento cirúrgico de pacientes portadores de metástases hepáticas de sarcomas de partes moles, com ênfase na identificação de fatores prognósticos e estudo de sobrevida. Método: Foi realizada análise retrospectiva de pacientes submetidos a ressecções hepáticas por sarcomas de partes moles metastáticos para fígado no Instituto Nacional de Câncer - MS entre 1992 e 2002. Fatores demográficos, características do tumor primário e dos tumores metastáticos, intervalo de surgimento de metástases, tipo de ressecção e resultados de sobrevida global e livre de doença, considerados a partir da operação para o tumor metastático foram considerados. Os dados obtidos foram analisados estatisticamente através dos softwares SPSS versão 8 e EpiInfo 2002. Resultados: O tumor primário era localizado no trato gastrintestinal em cinco pacientes, no retroperitônio em quatro e nos dois restantes no útero e no músculo deltóide. Leiomiossarcoma foi a linhagem histológica em 10 casos e dermatofibrossarcoma no caso restante, com grau de diferenciação tumoral G2 em seis casos e G3 em cinco. A extensão da ressecção hepática foi a segmentectomia em cinco pacientes, a lobectomia em quatro e as ressecções maiores em dois pacientes. Dois pacientes tiveram ressecções extra-hepáticas associadas. A ressecção foi completa em sete pacientes, persistindo doença residual macroscópica em quatro pacientes. Pela análise multivariada a sobrevida foi afetada pela radicalidade da ressecção, com 49 meses para as cirurgias completas e 15 meses para as incompletas $(\mathrm{p}<0,05)$ e pelo grau de diferenciação tumoral, com 49 meses para os tumores G2 e 15 meses para os G3 ( $<<0,0447)$. Conclusão: As ressecções hepáticas para metástases de sarcomas de partes moles podem aumentar a sobrevida destes pacientes, particularmente quando completas e para tumores de baixo grau de diferenciação (Rev. Col. Bras. Cir. 2006; 33(6): 380-386).
\end{abstract}

Descritores: Sarcoma; Neoplasias de tecidos moles; Hepatectomia; Metástase

\section{INTRODUÇÃO}

Os sarcomas de partes moles (SPM) com doença metastática estão associados à sobrevida curta. A mediana de sobrevida a partir do diagnóstico das metástases é de oito a 12 meses, podendo em 20 a $25 \%$ dos casos chegar a dois anos ${ }^{1,2}$. O valor da ressecção cirúrgica da metástase hepática dos SPM ainda não está definido na literatura, com poucas publicações abordando esta questão. Os resultados são controversos, quando comparados a hepatectomias para implantes secundários de carcinoma colorretal e de tumores neuroendócrinos, que estatisticamente, se associam a melhores índices de sobrevida ${ }^{3-6}$. Neste estudo, é analisada a experiência do Instituto Nacional de Câncer (INCA), com hepatectomias para tratamento de pacientes com sarcomas de partes moles metastáticos para o fígado.

\section{MÉTODO}

Foi realizada análise retrospectiva de pacientes submetidos a ressecções hepáticas por sarcomas de partes moles metastáticos para fígado no INCa entre 1992 e 2002. As informações contidas nos prontuários foram revisadas e utilizadas para este estudo. Fatores demográficos, características do tumor primário e dos tumores metastáticos, intervalo de surgimento das metástases, tipo de ressecção e resultados de sobrevida global e livre de doença, considerados a partir da cirurgia para o tumor metastático foram considerados. Pacientes com sarcomas hepáticos primários ou com invasão direta foram excluídos.

Para pacientes com múltiplas metástases hepáticas o diâmetro da maior delas foi considerado como o tamanho tumoral. Metástases sincrônicas foram referidas como aquelas cujo intervalo de tempo entre a detecção do tumor primário e o seu surgimento foi inferior a seis meses. As demais foram consideradas metacrônicas. As ressecções foram definidas como segmentectomias, lobectomias e ressecções maiores, quando além de lobectomia mais algum segmento hepático tenha sido ressecado. Ressecções extra-hepáticas associadas foram assinaladas quando presentes. As ressecções foram consideradas completas quando não persistiu doença

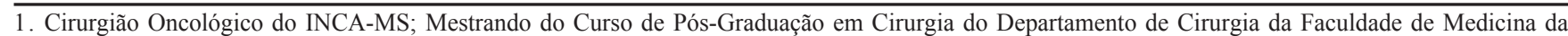
UFRJ.

2. Cirurgião Oncológico do INCA-MS

3. Chefe da Seção de Cirurgia Abdômino-Pélvica do INCA-MS.

4. Professor Adjunto do Departamento de Cirurgia da Faculdade de Medicina da UFRJ.
}

Recebido em 02/06/06

Aceito para publicação em 04/07/06

Conflito de interesses: nenhum

Fonte de financiamento: nenhuma

Trabalho realizado no Instituto Nacional de Câncer do Rio de Janeiro; Trabalho de conclusão do Curso de Pós-Graduação em Cirurgia, nível Mestrado, do Departamento de Cirurgia da Faculdade de Medicina da UFRJ. 
macroscópica e o estudo histopatológico da peça cirúrgica demonstrou margens livres.

A análise estatística dos dados obtidos foi realizada através dos softwares SPSS versão 8 e EpiInfo 2002. A análise de sobrevivência foi realizada pelo método de Kaplan-Meyer e as curvas de sobrevida foram comparadas pelo log-Rank test. Para as tabelas 2x2 realizou-se o teste de Fisher, Qui quadrado ou Qui quadrado corrigido por Yates. Os testes de Kruskal-Wallis, Man Whitney e t-student foram usados quando indicados. Significância estatística foi definida com um valor de $p<0,05$.

\section{RESULTADOS}

Dados Demográficos - Entre 1992 e 2002, 11 pacientes foram submetidos a ressecções hepáticas devido SPM metastáticos para fígado. A mediana de idade foi de 56 anos (30 - 75 anos) sendo sete pacientes do sexo feminino e quatro do sexo masculino (Tabela 1).

Características Tumorais - Em relação ao sítio primário, cinco pacientes apresentaram tumores no trato gastrintestinal e quatro no retroperitônio. Os dois restantes eram localizados no útero e no músculo deltóide. Os achados histopatológicos foram de leiomiossarcoma em 10 casos e dermatofibrossarcoma em um caso. Quanto ao grau de diferenciação, o tumor foi considerado G3 em cinco casos e G2 em seis.

A mediana de tempo entre a operação do tumor primário e o surgimento das metástases hepáticas foi de 28 meses (0- 287 meses), com três casos de metástases sincrônicas. A média do número de metástases foi de 1,8 (um a seis) por paciente, com tamanho médio de $9,7 \mathrm{~cm}$ (três a $18 \mathrm{~cm}$ ), e bilobar em três pacientes. Um paciente com tumor primário em retroperitônio apresentou metástase para pulmão e fígado (Tabela 2).

Tratamento - Os pacientes com sarcoma uterino e com sarcoma em músculo deltóide receberam radioterapia (RT) sobre a pelve e leito tumoral respectivamente. Quimioterapia (QT) neoadjuvante foi realizada em um paciente e adjuvante em outros seis. Esquemas baseados em doxorrubicina foram utilizados em todos estes pacientes.

Quatro pacientes foram submetidos a lobectomia, cinco a ressecções menores e os outros dois restantes realizaram ressecções hepáticas maiores. Ressecções simultâneas de sítios metastáticos extra-hepático ocorreram em dois pacientes. A ressecção foi completa em sete pacientes, persistindo doença macroscópica em quatro. Não houve persistência de doença residual microscópica em nenhum dos pacientes submetidos à ressecção completa (Tabela 3).

Sobrevida - Nenhuma morte operatória (até 30 dias do procedimento) ocorreu. A mediana de sobrevida global foi de 38 meses (seis a 63 meses), com sobrevida livre de doença de sete meses ( 0 a 29 meses). Quatro pacientes estão vivos até o momento de encerramento do trabalho, com sobrevida de 22, 40, 48 e 63 meses, todos com recidiva tumoral, dois dos quais já submetidos à nova ressecção tumoral após a ressecção hepática inicial (Tabela 4).
Tabela 1 - Fatores demográficos.

Idade (anos)

$\begin{array}{lr}\text { Mediana } & 56 \\ \text { Média } & 53 \\ \text { Variação } & 30-75\end{array}$

$\operatorname{Sexo}(n)$

$\begin{array}{ll}\text { Masculino } & 4 \\ \text { Feminino } & 7\end{array}$

Tabela 2 - Características Tumorais.

Sítio primário $(\mathrm{n})$

$\begin{array}{ll}\text { Retroperitônio } & 4 \\ \text { Estômago } & 3 \\ \text { Delgado } & 2 \\ \text { Outros } & 2\end{array}$

Tipo Histológico (n)

Leiomiossarcoma 10

Dermatofibrossarcoma 1

Grau Histológico (n)

$\begin{array}{ll}\text { G2 } & 6 \\ \mathrm{G} 3 & 5\end{array}$

Número de Metástases

Média $\quad 1,8$

Variação (1-6)

Localização

Unilateral (n) $\quad 8$

Tamanho (cm)

Bilateral 3

Média

Variação

Tempo de surgimento

Mediana (meses) 28

Sincrônico (n) 3

Metacrônico

Tabela 3 - Tratamento Cirúrgico.

Tipo de Ressecção

$\begin{array}{ll}\text { Segmentectomia } & 5 \\ \text { Lobectomia } & 4 \\ \text { Maior } & 2\end{array}$

Radicalidade

Curativa(R0) 7

Não Curativa (R2) 4

A análise univariada demonstrou significância estatística para predição de sobrevida, o grau de diferenciação tumoral $(p=0,028)$ e a radicalidade cirúrgica $(p=0,037)$. Idade, sexo, sítio tumoral, número de metástases, localização e tempo de surgimento, assim como tipo de cirurgia realizada e tratamento adjuvante associado, não foram fatores preditivos de sobrevida (Tabela 5). 
Tabela 4 - Dados clínicos e resultados de sobrevida.

\begin{tabular}{|c|c|c|c|c|c|c|c|c|c|}
\hline \multicolumn{2}{|c|}{ Pacienteld } & \multirow{2}{*}{$\frac{\text { Sexo }}{F}$} & \multirow{2}{*}{$\frac{\text { Primário }}{\text { Retroperit. }}$} & \multirow{2}{*}{$\frac{\text { Grau }}{\text { G3 }}$} & \multirow{2}{*}{$\frac{\text { Cir. }}{\geq \text { Lobec. }}$} & \multirow{2}{*}{$\frac{\text { Radical.* }}{\text { R2 }}$} & \multirow{2}{*}{$\frac{\text { QT\# }}{\text { Sim }}$} & \multirow{2}{*}{$\frac{\text { Sobv }+}{20}$} & \multirow{2}{*}{$\frac{\text { Status }}{\mathrm{MCD}}$} \\
\hline 1 & 41 & & & & & & & & \\
\hline 2 & 75 & F & Estômago & G2 & Seg. & $\mathrm{R} 0$ & Sim & 39 & MCD \\
\hline 3 & 62 & $\mathrm{~F}$ & Deltóide & G2 & $\geq$ Lobec. & $\mathrm{R} 0$ & Não & 48 & VCD \\
\hline 4 & 58 & M & Retroperit. & G3 & $\overline{\text { Seg. }}$ & $\mathrm{R} 0$ & Sim & 40 & $\mathrm{VCD}$ \\
\hline 5 & 44 & $\mathrm{~F}$ & Delgado & G2 & Seg. & R2 & Sim & 22 & VCD \\
\hline 6 & 56 & M & Retroperit. & G2 & $\geq$ Lobec. & R0 & Não & 49 & MCD \\
\hline 7 & 66 & $\mathrm{~F}$ & Retroperit. & G3 & Seg. & $\mathrm{R} 2$ & Sim & 06 & MCD \\
\hline 8 & 52 & M & Estômago & G2 & $\geq$ Lobec. & R0 & Sim & 38 & MCD \\
\hline 9 & 30 & M & Delgado & G3 & $\geq$ Lobec. & $\mathrm{R} 2$ & Não & 15 & MCD \\
\hline 10 & 44 & $\mathrm{~F}$ & Útero & G3 & $\geq$ Lobec. & $\mathrm{R} 0$ & Não & 13 & $\mathrm{MCD}$ \\
\hline 11 & 56 & $\mathrm{~F}$ & Estômago & $\mathrm{G} 2$ & $\overline{\text { Seg. }}$ & $\mathrm{R} 0$ & Sim & 63 & $\mathrm{VCD}$ \\
\hline
\end{tabular}

* Radical $=$ Radicalidade

\#QT = Quimioterapia

+ Sobv. = Sobrevida total

$V C D=$ Vivo com doença

$M C D=$ Morto com doença

Tabela 5 - Análise de Sobrevida.

$\mathrm{Na}$ análise multivariada o grau de diferenciação tumoral, com mediana de sobrevida de 49 meses para os tumores G2 e 15 meses para os tumores G3 $(p<0,0447)$ e a radicalidade da ressecção, com mediana de sobrevida de 49 meses para os tumores completamente ressecados e 15 meses para os pacientes que mantiveram doença residual $(p<0,05)$, permaneceram estatisticamente significantes quando calculados pelo log Rank test.

\section{DISCUSSÃO}

Os sarcomas de partes moles são tumores raros o que resulta em poucos estudos sobre o tema e conseqüentemente em um entendimento ainda impreciso de todos os aspectos de sua evolução biológica e das estratégias para o seu controle. Uma pobreza de informações ainda maior é observada quanto ao manejo de pacientes portadores de metástases hepáticas deste tipo de tumor. Até o momento não existem estudos que tragam informações precisas sobre a adequação do tratamento cirúrgico neste grupo de pacientes. Os estudos disponíveis apresentam um número pequeno e heterogêneo de pacientes, com seus dados coletados, na maioria das vezes, de maneira retrospectiva e não controlada.

O presente estudo compreende uma casuística de 11 pacientes selecionados de um universo de 66587 pacientes portadores de neoplasias tratados no INCA durante o período do estudo, dos quais 1531 (2,29\%) eram portadores de SPM. Observa-se assim que a indicação de ressecção hepática para tratamento de metástases de SPM foi altamente seletiva, com apenas 11 casos em 10 anos. Nosso principal objetivo foi tentar determinar que grupo de pacientes pode se beneficiar destas ressecções, que, até o momento, tem indicação restrita. Sexo, idade, localização do sítio primário, apresentação (sincrônico ou metacrônico e lobar ou bilobar) e número de metástases não foram estatisticamente significantes para predição de sobrevida, em concordância com dados da literatura

\begin{tabular}{|c|c|c|}
\hline Variável & n & Valor de $p$ \\
\hline \multicolumn{3}{|l|}{ Sexo } \\
\hline Masc & 4 & 0,57 \\
\hline Fem & 7 & \\
\hline \multicolumn{3}{|l|}{ Idade } \\
\hline$\leq 56$ & 7 & 0,85 \\
\hline$>56$ & 4 & \\
\hline \multicolumn{3}{|l|}{ Sítio Primário } \\
\hline TGI* & 5 & 0,82 \\
\hline Outros & 6 & \\
\hline \multicolumn{3}{|l|}{ Apresentação } \\
\hline Sincrônico & 3 & 1,00 \\
\hline Metacrônico & 8 & \\
\hline \multicolumn{3}{|l|}{ Apresentação } \\
\hline Lobar & 8 & 0,63 \\
\hline Bilobar & 3 & \\
\hline \multicolumn{3}{|l|}{ ILD (Mediana)** } \\
\hline$<28$ meses & 5 & 0,20 \\
\hline$\geq 28$ meses & 6 & \\
\hline \multicolumn{3}{|l|}{ Ext. Hepatectomia } \\
\hline$<$ Lobectomia & 5 & 0,71 \\
\hline$\geq$ Lobectomia & 6 & \\
\hline \multicolumn{3}{|l|}{ Ressec. Assoc. } \\
\hline Sim & 2 & 0,31 \\
\hline Não & 9 & \\
\hline \multicolumn{3}{|l|}{ QT Adjuvante } \\
\hline $\operatorname{Sim}$ & 5 & 0,43 \\
\hline Não & 6 & \\
\hline \multicolumn{3}{|c|}{ Grau de Diferenciação } \\
\hline G2 & 6 & 0,028 \\
\hline G3 & 5 & \\
\hline \multicolumn{3}{|l|}{ Radicalidade } \\
\hline R0 & 7 & $\mathbf{0 , 0 3 7}$ \\
\hline $\mathrm{R} 2$ & 4 & \\
\hline
\end{tabular}


7-11. A extensão da cirurgia hepática e a existência ou não de ressecções associadas, de igual maneira não interferiram na sobrevida.

Em 1977, Russell ${ }^{12}$ propôs uma classificação para os sarcomas introduzindo o conceito de gradação histológica, aplicável para os sarcomas em adultos. No seu estudo, grau histológico foi o fator prognóstico mais importante entre os analisados. Desde então, vários sistemas de gradação têm sido propostos. Atualmente os dois sistemas mais utilizados são o do National Cancer Institute (NCI), proposto por Costa $^{13}$ em 1984 e modificado em $1990^{14}$ e o da Fédération Nationale des Centres de Lutte Contre le Cancer (FNCLCC) desenvolvido pela French Federation of Cancer Centers Sarcoma Group ${ }^{15}$.

No INCA utiliza-se o sistema de gradação do NCI, para classificação dos sarcomas. No nosso estudo foram encontrados seis pacientes com Grau 2 de diferenciação tumoral e cinco pacientes com Grau 3. Os seis pacientes com tumores Grau 2 apresentaram sobrevida mediana de 49 meses enquanto a mediana de sobrevida para pacientes com tumores Grau 3 foi de 15 meses ( $<<0,047)$. Diferentemente do encontrado em nossa série, o grau de diferenciação não foi identificado como fator independente para sobrevida nas demais séries envolvendo a avaliação de sobrevida pós-ressecção de metástases hepáticas de SPM. Chen ${ }^{9}$ no seu estudo envolvendo também 11 pacientes, não descreve qual foi o sistema empregado para gradação em sua população. Para análise estatística comparou a sobrevida entre os sarcomas de baixo Grau (considerados Grau 1) e os de alto Grau (considerados Grau 2 e Grau 3) não obtendo diferença estatisticamente significante. Lang ${ }^{10}$ avaliou ressecções hepáticas por metástases de leiomiossarcoma em 34 pacientes, empregando um sistema de gradação com quatro subdivisões. Para efeito de análise, dividiu os tumores em baixo Grau (Grau 1 e 2) e alto Grau (grau 3 e 4). Todos os pacientes, menos um, tinham tumores considerados de baixo grau, o que não permitiu conclusões quanto à importância do Grau de diferenciação na sobrevida pós-ressecção. A maior série envolvendo ressecções hepáticas para SPM, foi relatada por DeMatteo ${ }^{11}$ no Memorial Sloan Kettering Cancer Center (MSKCC). O sistema de gradação utilizado por este grupo utiliza para sua determinação o grau de celularidade, diferenciação e vascularização, pleomorfismo nuclear, número de mitoses por campo de grande aumento e quantidade de necrose, diferenciando-se, portanto, dos sistemas utilizados pela FNCLCC e pelo NCI. Nesta série, envolvendo 56 pacientes, não é feito relato sobre os resultados referentes ao grau de diferenciação tumoral quanto ao impacto na sobrevida.

Grau de diferenciação histológica também tem sido apontado como um dos principais fatores para o desenvolvimento de metástases à distância. Coindre ${ }^{16} \mathrm{em}$ uma série de 1240 pacientes com SPM, relatou risco de desenvolvimento de metástases à distância 7,8 vezes maior para os pacientes com tumores Grau 3 quando comparados aos de Grau 1. Zagars ${ }^{17}$ em estudo realizado no MD Anderson Cancer Center, de maneira similar, encontrou risco 22.5 vezes maior em uma população de 225 pacientes. Pister $^{18}$ estudando uma população de 1041 pacientes encontrou risco relativo de desenvolvimento de metástase a distância 4,3 vezes maior para os sarcomas de alto grau quando comparados aos de baixo grau de diferenciação. A maioria das metástases a distância surge de tumores de alto grau de diferenciação, porém um subgrupo de pacientes desenvolve metástases a partir de tumores de baixo grau, podendo representar tumores de características biológicas mais agressivas ou mesmo nichos de pior grau de diferenciação em tumores predominantemente bem diferenciados. A sobrevida pós-metástases neste grupo de pacientes ainda não é bem avaliada. Nas séries de Coindre, ${ }^{16}$ Zagars ${ }^{17}$ e Pister, ${ }^{18}$ uma vez ocorrendo metástases à distância, o grau de diferenciação não se apresentou como um fator prognóstico. No entanto, em estudos de sobrevida pós-ressecção de metástases pulmonares de sarcomas, Bilingsley ${ }^{19}$ encontrou melhor sobrevida para pacientes com tumores de baixo grau ( $p=$ 0.02 ), fato também observado no estudo de Van Geel ${ }^{20}$. Uma conclusão sobre o impacto do grau de diferenciação tumoral, na sobrevida pós-ressecção das metástases hepáticas neste tipo de tumor, torna-se difícil pelo pequeno número de pacientes que se submetem à cirurgia de resgate e também pela não padronização dos sistemas empregados para gradação, de tal maneira que um tumor considerado de um determinado grau por um sistema pode ser diferentemente graduado quando submetido à outra metodologia de classificação.

No nosso estudo foi também avaliado o impacto do intervalo entre o surgimento das metástases e o diagnóstico do tumor primário (intervalo livre de doença - ILD), como possível fator prognóstico. Além da avaliação referente a sincrônico ou metacrônico (intervalo de seis meses), foi analisado um intervalo livre de doença referente à mediana do tempo de surgimento das metástases, sendo este valor correspondente a 28 meses. No nosso grupo esta variável não mostrou valor prognóstico para sobrevida pós-ressecção, diferentemente dos resultados encontrados por DeMatteo que, analisando especificamente pacientes submetidos à ressecção hepática por SPM, encontrou sobrevida mediana de 61 meses nos pacientes com ILD maior que dois anos contra 26 meses para os pacientes com ILD inferior a dois $\operatorname{anos}(p=0.002)$.

O intervalo livre de doença tem sido apontado como um importante fator preditivo de resultado em neoplasias como carcinomas colorretais, melanoma maligno, carcinomas de mama e renal ${ }^{4,21-25}$. O papel desta variável após o desenvolvimento de metástases em SPM ainda não é completamente entendido. Vezeridis ${ }^{26}$ em um grupo de 242 pacientes com recidiva local e a distância de SPM, encontrou sobrevida mediana de 7,5, 10,9 e 22,1 meses, para pacientes com ILD de zero, menor que 12 e maior que 12 meses respectivamente. Stojadinovic ${ }^{27}$ analisou 2.123 pacientes com SPM tratados no MSKCC entre 1982 e 1999 e 788 (37\%) desenvolveram recidiva local e/ou à distância durante o seguimento. A mediana de sobrevida foi significativamente melhor para ILD maiores que 12 meses nos tumores de tronco e extremidades localmente recorrentes (50,4 v 29,8 me- 
ses; $p<0.01)$ e nos tumores de retroperitônio $(49,9 v 9,9$ meses; $p=0.03$ ). Ainda neste estudo foi observado que a probabilidade de se conseguir uma nova ressecção efetiva após a recidiva, foi de $2 \%$ nos pacientes que recidivaram em um período menor que seis meses após a ressecção em tumores primários de alto grau, profundos e maiores que 5 $\mathrm{cm}$. Nos pacientes com tumores com as mesmas características, porém com um ILD maior que três anos, esta probabilidade subia para $30 \%$.

A possibilidade de ressecção completa R0 é condição primordial a ser considerada na indicação de cirurgia para tratamento de metástases hepáticas de SPM, o que, no entanto, pode não se concretizar durante o ato operatório, e que ocorreu com quatro dos nossos pacientes, que permaneceram com doença residual macroscópica após a ressecção hepática. A sobrevida mediana foi significativamente menor nos pacientes com doença residual, com sobrevida de 15 meses contra 49 meses para os pacientes totalmente ressecados $(p<0.05)$. Nos estudos de $\mathrm{Chen}^{9} \mathrm{e}$ Lang ${ }^{10}$ resultados similares foram encontrados quando comparados pacientes sem doença residual com pacientes com doença residual macroscópica ou microscópica, após ressecções hepáticas. No maior estudo envolvendo ressecções hepáticas de SPM metastáticos, DeMatteo ${ }^{11}$ considerou ressecções completas aquelas em que toda a doença macroscópica foi ressecada, independente da persistência de doença microscópica. Neste estudo envolvendo 56 pacientes, 42 (75\%) tiveram margens negativas e 14 tiveram margens microscópicas positivas, com sobrevida mediana de 39 meses e de 25 meses respectivamente, diferença não estatisticamente significante. Estes pacientes foram considerados como tendo ressecções completas e quando comparados com aqueles nos quais houve persistência de doença macroscópica ou não submetidos ao tratamento operatório, houve um ganho significativo em sobrevida $(p<0.0001)$. Apesar de não haver diferença estatisticamente significante em sobrevida com relação à presença ou não de margens microscópicas positivas, a taxa de recorrência após ressecção hepática foi de $84 \%$ (47 pacientes). As informações precisas sobre as recorrências foram disponíveis em 39 destes 47 pacientes, tendo sido observada recidiva hepática em $56 \%$, provavelmente devido à doença hepática microscópica residual, como sugerido pelo próprio autor. As margens microscopicamente comprometidas estão intimamente ligadas à recidiva local e em alguns estudos com sobrevida relacionada à doença, em pacientes submetidos a ressecções para sarcomas primários. Conclusões precisas sobre o impacto de margens positivas após ressecções hepáticas por metástases de SPM tornam-se difíceis pela pequena quantidade de estudos abordando esta questão. Considerando-se os dados envolvendo os resultados pós-ressecção para tumores primários com claro impacto negativo das margens microscópicas comprometidas nas taxas de controle local e na sobrevida relacionada à doença, ${ }^{17,18,27,28}$ torna-se razoável considerar que esta mesma variável apresente, igualmente, um impacto negativo após hepatectomias para metástases de SPM.

Resultados com quimioterapia para sarcomas de partes moles são desapontadores. Taxas de resposta variando entre 20 e $30 \%$ podem ser conseguidas com doxorrubicina ou ifosfamida, os dois principais agentes utilizado ${ }^{1 .}$ No nosso estudo sete pacientes foram submetidos ao tratamento quimioterápico em algum momento do curso de sua doença. Doxorrubicina foi utilizado em todos os esquemas empregados, mas não foi observado qualquer impacto na sobrevida. Associações de drogas têm sido largamente testadas,assim como novos agentes terapêuticos, mas apesar de algumas combinações apresentarem taxas de respostas superiores quando comparadas à associação entre doxorrubicina e ifosfamida, não se mostrou ganho de sobrevida efetivo, com taxas significativamente diferentes em toxicidade ${ }^{29-32}$.

No nosso estudo nenhum paciente teve diagnóstico de tumor estromal gastrintestinal (GIST). Até o ano de 2000 não havia critérios definidos para o diagnóstico de GIST, de modo que a maioria dos tumores mesenquimais era classificada como leiomiomas ou leiomiossarcomas. Com o advento da microscopia eletrônica e melhor desenvolvimento de técnicas imunohistoquímicas, observou-se que estes tumores, na sua maioria, não apresentavam características de diferenciação para músculo liso, tratando-se na verdade de uma categoria distinta de tumores, chamada tumores do estroma gastrintestinal, (Gastrointestinal Stromal TumoursGIST), caracterizados pela expressão do receptor tirosina kinase c-KIT e por taxas de resposta significativas ao tratamento com mesilato de imatinibe $e^{33,34,35}$.Estes tumores apresentam, no entanto, comportamento clínico e taxas de sobrevivência semelhantes, ou mesmo piores do que as dos leiomiossarcomas intra-abdominais, quando não submetidos ao tratamento com imatinibe. ${ }^{36-39}$. Atualmente sabe-se que cerca de $80 \%$ dos tumores de origem mesenquimal do trato gastrintestinal correspondem a GIST, com apenas $15 \%$ constituídos verdadeiramente por tumores de origem muscular ${ }^{35}$. Na nossa casuística, cinco pacientes tiveram tumores localizados em estômago e intestino delgado. Três deles morreram antes de 2000 e os outros dois, ao fim do estudo, não haviam sido pesquisados para GIST, e não utilizaram terapêutica com imatinibe.

Pacientes com sarcomas de partes moles avançados são habitualmente tratados com cirurgia, quimioterapia ou cuidados paliativos. Terapias ablativas, quimioembolização e transplantes como tratamento para este grupo de pacientes têm sido usados, mas ainda como tratamentos investigacionais ${ }^{2}$. No nosso estudo, nenhum paciente foi submetido a qualquer destas modalidades terapêuticas.

Os resultados deste trabalho sugerem que as ressecções hepáticas para metástases de sarcomas de partes moles podem aumentar a sobrevida destes pacientes, particularmente quando completas e para tumores de baixo grau de diferenciação. 


\begin{abstract}
Background: The objective of this study was to evaluate the surgical treatment of patients with liver metastasis of soft tissue sarcomas, with emphasis on the identification of prognostic factors and survival. Methods: This is a retrospective analysis of liver resections for metastatic soft tissue sarcomas performed at National Institute of Cancer in Rio de Janeiro, between 1992 and 2002. Demographic factors, characteristics of the primary and metastatic tumors, disease free interval, type of liver resections, overall survival and disease free interval after liver resections were studied. Statistical analysis were performed with version 8 SPSS and EpiInfo 2002. Results: In five patients the primary tumor was localized at the gastrointestinal tract, in four at the retroperitoneum, in one at the uterus and in one at the deltoid muscle. Ten of the tumors were leiomyosarcomas and one was a dermatofibrosarcoma. Six patients had G2 differentiation grade tumors and the other five had G3 tumors. Five patients underwent to segmentectomies, four to lobectomies and two to major hepatic resections. Two patients underwent to extra-hepatic associated resections. Complete resection was accomplished in seven patients. Multivariate analysis showed that overall survival was directly affected by complete resection with a 49-month survival for R0 resections compared to a 15-month survival for patients with residual disease ( $p<0.05)$. Similarly, patients with $G 2$ tumors had a 49-month survival compared to a 15-month survival for patients with G3 tumors ( $p<0.0447)$. Conclusion: Liver resections for hepatic metastasis of soft tissue sarcomas can improve overall survival particularly for patients with complete resection (R0) and for low grade tumors.
\end{abstract}

Key Words: Sarcoma; Soft Tissue Neoplasms; Hepatectomy; Metastases.

\section{REFERÊNCIAS}

1. Brennan MF, Singer S, Maki RG, O’SullivanB. Soft tissue sarcoma. In: Devita VT, Hellman S, Rosenberg SA. Cancer: Principles and Practice of Oncology, $7^{\text {th }}$ Edition. Philadelphia: Linppicott-Raven Publishers, 2005: 1581-1837.

2. Abdalla EK, Pisters PWT. Metastasectomy for limited metastases from soft tissue sarcoma. Curr Treat Options Oncol. 2002 Dec;3(6):497-505.

3. Gayowski TJ, Iwatsuki S, Madariaga JR, Selby R, Todo S, Irish W, Starzl TE. Experience in hepatic resection for metastatic colorectal cancer: analysis of clinical and pathologic risk factors. Surgery. 1994 Oct;116(4):703-10; discussion 710-1. Comment in: Surgery. 1996 Jan;119(1):118-20.

4. Fong Y, Fortner J, Sun RL, Brennan MF, Blumgart LH. Clinical score for predicting recurrence after hepatic resection for metastatic colorectal cancer: analysis of 1001 consecutive cases. Ann Surg. 1999 Sep;230(3):309-18; discussion 318-21.

5. Que FG, Nagorney DM, Batts KP, Linz LJ, Kvols LK. Hepatic resection for metastatic neuroendocrine carcinomas. Am J Surg. 1995 Jan;169(1):36-42; discussion 42-3.

6. McEntee GP, Nagorney DM, Kvols LK, Moertel CG, Grant CS. Cytoreductive hepatic surgery for neuroendocrine tumors. Surgery. 1990 Dec;108(6):1091-6.

7. Ng EH, Pollock RE, Romsdahl MM. Prognostic implications of patterns of failure for gastrointestinal leiomyosarcomas. Cancer. 1992 Mar 15;69(6):1334-41.

8. Hafner GH, Rao U, Karakousis CP. Liver metastasis from soft tissue sarcomas. J Surg Oncol. 1995 Jan;58(1):12-6.

9. Chen H, Pruitt A, Nicol TL, Gorgulu S, Choti MA. Complete hepatic resection of metastases from leiomyosarcoma prolongs survival. J Gastrointest Surg. 1998 Mar-Apr;2(2):151-5.

10. Lang H, Nussbaum KT, Kaudel P, Fruhauf N, Flemming P, Raab R. Hepatic metastases from leiomyosarcoma. A single-center experience with 34 liver resections during a 15 -year period. Ann Surg. 2000 Apr;231(4):500-5.

11. DeMatteo RP, Shah A, Fong Y, Jarnagin WR, Blumgart LH, Brennan MF. Results of hepatic resection for sarcoma metastatic to liver. Ann Surg. 2001 Oct;234(4):540-7; discussion 547-8.

12.. Russell WO, Cohen J, Enzinger F, Hajdu SI, Heise H, Martin RG, Meissner W, Miller WT, Schmitz RL, Suit HD. A clinical and pathological staging system for soft tissue sarcomas. Cancer. 1977 Oct;40(4):1562-70.
13. Costa J, Wesley RA, Glatstein E, Rosenberg SA. The grading of soft tissue sarcomas. Results of a clinicohistopathologic correlation in a serie of 163 cases. Cancer. 1984 Feb 1;53(3):53041.

14. Costa J. The grading and staging of soft tissue sarcomas, in Fletcher CDM, McKee PH: Pathobiology of Soft Tissue Tumours. Edinburgh, UK, Churchill Livingstone, 1990, pp 221-238.

15. Trojani M, Contesso G, Coindre JM, Rouesse J, Bui NB, de Mascarel A, Goussot JF, David M, Bonichon F, Lagarde C. Softtissue sarcomas of adults: Study of pathological prognostic variables and definitions of a histopathological grading system. Int J Cancer. 1984 Jan 15;33(1):37-42.

16. Coindre JM, Terrier P, Guillou L, Le Doussal V, Collin F, Ranchere D, Sastre X, Vilain MO, Bonichon F, N'Guyen Bui B. Predictive value of grade for metastasis development in the main histologic types of adult soft tissue sarcomas. A study of 1240 patients from the French Federation of Cancer Centers Sarcoma Group. Cancer. 2001 May 15;91(10):1914-26.

17. Zagars GK, Ballo MT, Pisters PW, Pollock RE, Patel SR, Benjamin RS, Evans HL. Prognostic factors for patients with localized soft-tissue sarcoma treated with conservation surgery and radiation therapy. An analysis of 1225 patients. Cancer. 2003 May 15;97(10):2530-43.

18. Pisters PWT, Leung DHY, Woodruff, Shi W, Brennan MF. Analysis of prognostic factors in 1041 patients with localized soft tissue sarcomas of the extremities. J Clin Oncol. 1996 May;14(5):1679-89.

19. Billingsley KG, Burt ME, Jara E, Ginsberg RJ, Woodruff JM, Leung DH, Brennan MF. Pulmonary metastases from soft tissue sarcoma. Analysis of patterns of disease and posmetastasis survival. Ann Surg. 1999 May;229(5):602-10; discussion 610-2.

20. van Geel AN, Pastorino U, Jauch KW, Judson IR, van Coevorden F, Buesa JM, Nielsen OS, Boudinet A, Tursz T, Schmitz PI. Surgical treatment of lung metastases: The European Organization for Research and Treatment of Cancer-Soft Tissue and Bone Sarcoma Group study of 255 patients. Cancer. 1996 Feb 15;77(4):675-82.

21. Dong XD, Tyler D, Johnson JL, DeMatos P, Seigler HF. Analysis of prognosis and disease progression after local recorrence of melanoma. Cancer. 2000 Mar 1;88(5):1063-71.

22. Barth A, Wanek LA, Morton DL. Prognostic factors in 1521 melanoma patients with distant metastases. J Am Coll Surg. 1995 Sep;181(3):193-201. 
23. Fisher B, Anderson S, Fisher ER, Redmond ScD C, Wickerham DL, Wolmark N. Significance of ipsilateral breast tumour recorrence after lumpectomy. Lancet. 1991 Aug 10;338(8763):327-31.

24. Veronesi U, Salvadori B, Luini A, Greco M, Saccozzi R, del Vecchio M, Mariani L, Zurrida S, Rilke F. Breast conservation is a safe method in patients with small cancer of the breast. Longterm results of three randomised trials on 1973 patients. Eur J Cancer. 1995 Sep;31A(10):1574-9. Comment in: Eur J Cancer. 1995 Sep;31A(10):1567-9.

25. Kavolius JP, Mastorakos DP, Pavlovich C, Russo P, Burt ME, Brady MS. Resection of metastatic renal cell carcinoma. J Clin Oncol. 1998 Jun;16(6):2261-6.

26. Vezeridis MP, Moore R, Karakousis CP. Metastatic patterns in soft-tissue sarcomas. Arch Surg. 1983 Aug;118(8):915-8.

27. Stojadinovic A, Leung DHY, Allen P. Lewis JJ, Jaques DP, Brennan MF. Primary adult soft tissue sarcoma: Time-dependent influence of prognostic variables. J Clin Oncol. 2002 Nov 1;20(21):4344-52.

28. Dickinson IC, Whitwell DJ, Battistuta D, Thompson B, Strobel N, Duggal A, Steadman P. Surgical margin and its influence on survival in soft tissue sarcoma. ANZ J Surg. 2006 Mar;76(3):104-9.

29. Spira AI, Ettinger DS. The use of chemotherapy in soft-tissue sarcomas. Oncologist. 2002;7(4):348-59.

30. Von Burton G, Rankin C, Zalupski MM, Mills GM, Borden EC, Karen A. Phase II trial of gemcitabine as first line chemotherapy in patients with metastatic or unresectable soft tissue sarcoma. Am J Clin Oncol. 2006 Feb;29(1):59-61.

31. Hartmann JT, Oechsle K, Huober J, Jakob A, Azemar M, Horger M, Kanz L, Bokemeyer C. An open label, non-comparative phase II study of gemcitabine as salvage treatment for patients with pretreated adult type soft tissue sarcoma Invest New Drugs. 2006 May;24(3):249-53.

32. Suppiah R, Wood L, Elson P, Budd GT. Phase I/II study of docetaxel, ifosfamide, and doxorubicin in advanced, recurrent, or metastatic soft tissue sarcoma (STS). Invest New Drugs. 2006 Nov;24(6):509-14.

33. Sircar K, Hewlett BR, Huizinga JD, Chorneyko K, Berezin I, Riddell RH. Interstitial cells of Cajal as precursors of gastrointestinal stromal tumors. Am J Surg Pathol. 1999 Apr;23(4):377-89.
34. Hirota S, Isozaki K, Moriyama Y, Hashimoto K, Nishida T, Ishiguro S, Kawano K, Hanada M, Kurata A, Takeda M, Muhammad Tunio G, Matsuzawa Y, Kanakura Y, Shinomura Y, Kitamura Y. Gain-of-function mutations of c-kit in human gastrointestinal stromal tumors. Science. 1998 Jan 23;279(5350):577-80.

35. Dimetri GD. Gastrointestinal Stromal Tumors In: Devita VT, Hellman S, Rosenberg SA. Cancer: Principles and Practice of Oncology, 7th Edition. Philadelphia: Linppicott-Raven Publishers, 2005: 1050-60.

36. Plaat BE, Hollema H, Molenaar WM, Torn Broers GH, Pijpe J, Mastik MF, Hoekstra HJ, van den Berg E, Scheper RJ, van der Graaf WT. Soft tissue leiomyosarcomas and malignant gastrointestinal stromal tumors: differences in clinical outcome and expression of multidrug resistance proteins. J Clin Oncol. 2000 Sep 15;18(18):3211-20.

37. DeMatteo RP, Lewis JJ, Leung D, Mudan SS, Woodruff JM, Brennan MF. Two hundred gastrointestinal stromal tumors: recurrence patterns and prognostic factors for survival. Ann Surg. 2000 Jan;231(1):51-8.

38. Shima Y, Horimi T, Ishikawa T, Ichikawa J, Okabayashi T, Nishioka Yet al. Aggressive surgery for liver metastases from gastrointestinal stromal tumors. J Hepatobiliary Pancreat Surg. 2003;10(1):77-80.

39. Nunobe S, Sano T, Shimada K, Sakamoto Y, Kosuge T. Surgery including liver resection for metastatic gastrointestinal stromal tumors or gastrointestinal leiomyosarcomas. Jpn J Clin Oncol. 2005 Jun;35(6):338-41. Epub 2005 May 31.

Como citar este artigo:

Silva RG, Paiva RKS, Santos CER, Melo ELR, Dias JA, Bravo Neto GP. Ressecção de metástase hepática de sarcomas de partes moles. Rev Col Bras Cir [periódico na Internet]. 2006 Nov-Dez;33(6). Disponível em URL: www.scielo.br/rcbc

Endereço para correspondência: Guilherme Pinto Bravo Neto

Rua Severiano da Fonseca, 97

21931-220- Rio de Janeiro - RJ 